\title{
Teaching the Placebo Effect
}

\author{
Javier Garcia
}

Received: 15 June 2014 / Accepted: 15 September 2014 / Published online: 21 October 2014

(C) Academic Psychiatry 2014

To the Editor:

In a letter to the editor in the April 2014 issue of Academic Psychiatry [1], Shaligram et al. described a pilot course focused on teaching brief psychotherapeutic interventions to residents, for use during a 15 -min medication management appointment. As a psychiatrist who works at a teaching hospital supervising residents for medication management and psychotherapy, I was impressed with their aim to integrate teaching of these important skills into standard practice.

In reading this letter, however, I felt that they left out one additional impact that their instructional technique might be having. Use of brief therapeutic interventions could enhance the therapeutic alliance. They could also be used to intentionally trigger the placebo effect.

A distinction should be made between a placebo and placebo effect. A placebo is often described as an "inert substance"; however, the placebo effect goes beyond the use of placebo; it is that aspect of treatment that is not attributable to specific pharmacologic or physiologic properties. Whether we are using an active medication or a validated form of therapy, the placebo effect probably accounts for more of our therapeutic effect than we would care to admit. Krupnick et al. showed a robust association between therapeutic alliance and improvement in both drug and placebo condition in a National Institute of Mental Health study [2]. A recent meta-analysis involving 200 research reports and more than 14,000 treatments found a strong correlation between therapeutic alliance and positive outcomes [3]. Many factors help trigger the placebo effect. The ones supported most frequently by empirical evidence include (1) positive expectancy on the part of the patient, (2) positive belief on the part of the health care professional, and (3) quality of patient-physician relationship or therapeutic alliance [4]. These are the factors we can teach to our learners to maximize their effectiveness.

Unfortunately, the placebo effect often invokes a negative response among practitioners, perhaps because it is associated

J. Garcia $(\bowtie)$

Richmond University Medical Center, Staten Island, NY, USA

e-mail: jvrgrc@yahoo.com with charlatanry or because researchers and pharmaceutical companies often view the placebo as an obstacle to overcome to prove the effectiveness of their drugs or interventions. As clinicians and teachers, we should not be trying to minimize, control, or select out the placebo effect; instead, we should be trying to maximize it and use it.

Teaching about the placebo effect goes beyond simply understanding the psychological component of psychiatric illnesses. It extends to the manner in which we engage patients, the words we use, our office environments, and even the kind of information we provide in pamphlets. Indeed, the 2002 National Institute of Health placebo conference emphasized the importance of identifying the qualities of placebo enhancing practitioners. As summarized by Brody [4], some of these qualities include exhibiting caring, empathy, sensitivity, and ability to adapt medical care goals to patient needs and values. They also provide encouragement to patients in decision making; they were perceived as warm, friendly, and trustworthy. In addition, they exuded confidence, enthusiasm, and willingness to be reassuring [5].

Shaligram et al. showed us that we can incorporate these key elements even into 15 -min visits. Thus, I think it is time for us as psychiatrists and teachers to embrace the placebo effect. In fact, I think it is time to officially include it as part of the psychiatry curriculum.

\section{References}

1. Shaligram D, Pearlmutter M, Nestelbaum Z, Brown W. Acad Psychiatry. 2014;38:242-3.

2. Krupnick JL, Sotsky SM, Simmens S, et al. The role of the therapeutic alliance in psychotherapy and pharmacotherapy outcome: findings in the National Institute of Mental Health Treatment of Depression Collaboration Research Program. J Consult Clin Psychol. 1996;64(3):532-9.

3. Del Re Fluckiger C, Wampold AC, Znoj BE, et al. How central is the alliance in psychotherapy? A multi-level longitudinal meta-analysis. J Couns Psychol. 2012;59(1):10-7.

4. Brody H. Placebo response, sustained partnership, and emotional resilience in practice. J Am Board Fam Pract. 1997;10(1):72-4.

5. Benson H, Epstein MD. The placebo effect-a neglected asset in the care of patients. JAMA. 1975;232:1225-7. 